\title{
Editorial: neonatal pain
}

Volume 3 Issue I - 2015

\author{
Khaled El-Atawi \\ Consultant Neonatologist, Latifa Hospital, UAE
}

new-born individualized development care and assessment program

Evidences from the recent observational and experimental studies in neonatal pain have debunked the myth that neonates are desensitised to pain and lack long-term memory about the pain. ${ }^{1}$ One of the popular hypothesis about neonatal desensitization to pain has specified that, owing to the cortical immaturity, neonates cannot perceive pain like infants, older children and adults. However; evidence based studies have elaborated that humans have an innate ability to perceive pain and to respond to the noxious stimuli. The fetus has the ability to perceive pain from 21 weeks of gestation while neonates perceive pain like an adult. Signs of a neonate experiencing pain include increased heart rate, blood cortisol, and beta-endorphin in response to activation of their autonomous nervous system. ${ }^{2}$

\section{The core issue- pre-term birth}

Epidemiological data for year 2010 showed that about $11.1 \%$ of all live births worldwide were pre-term. ${ }^{3,4}$ An increasing number of pre-term birth and associated neonatal care and life-saving procedures have significantly increased the episodes of pain among neonates. ${ }^{5}$

Neonates exposed to painful stimuli are highly susceptible to acute, and long-term consequences of pain that negatively affect their psychological, emotional, behavioural, and cognitive development. ${ }^{6}$ Animal model studies for neonatal pain have showed that persistent or repetitive pain increases apoptosis of neurone that could results in anxiety-disorders later in life. ${ }^{7}$ In humans, the pain transmission and pain modulation pathways usually start developing from $22 \mathrm{nd}$ week of gestation and are fully developed till the age of 2 months. ${ }^{8}$ It has been found that neonates subjected to nociceptive stimuli during this vulnerable period undergo long-term epigenetic changes, which affect their brain, neurodevelopment, pain modulation, and pain reactivity into adulthood. ${ }^{9}$ Studies reported a strong association between neonatal pain/stress and altered IQ at age of seven years, probably due to irreversible changes in brain microstructure..$^{10}$ In a longitudinal cohort study of neonates (from birth to school-age), it was found that painful experience in neonatal period is linked to the altered spontaneous oscillatory brain activity, which correlates with the visual problem-solving abilities later in life. ${ }^{11}$ Procedural pain in preterm neonates is also found to be closely related to cognitive and learning disorders that surface later in life. ${ }^{12}$ Therefore, for humanitarian reason it is very important to develop an effective and safe pain management strategy for treating neonatal pain and distress.

\section{Innovative pain management strategies}

Practice of pain management strategies remains an unrecognized aspect of neonatal care in most of NICUs. To overcome such clinical barrier the International evidence-based group for neonatal pain developed a consensus on clinical practices guidelines for pain management among neonates. These guidelines highlighted the importance of recognition of site/ location of pain in neonates, avoidance of noxious stimuli, and use of environmental, behavioural, and pharmacological interventions to minimize the pain. ${ }^{13}$ The Consensus statement also acknowledged the use of neonate-specific care plan and analgesic protocol to be used in different clinical situations. Clinical practice guidelines Pain Study Group of the Italian Society of Neonatology focus on analgesia and sedation protocol for neonates undergoing invasive procedures in NICU. ${ }^{14}$

The WHO developed strategies primarily focus on effective developmental care practices in neonates like positioning, clustering of nursery care activities, modification of external stimuli, and New-born individualized development care and assessment program (NIDCAP). NIDCAP focuses on; protected sleep, pain and stress assessment and management, developmentally supportive activities of daily diving, family-centered care, and creating a healing environment. ${ }^{15}$ Most of the pain management strategies that have been used remain unsuccessful in managing pain and distress among neonates, mainly due to the inability of a neonate to express pain. Crying is the only expression neonates have to an inevitable expression for hunger, discomfort, physiological pain, or distress which makes it difficult for the care-giver to identify and assess the sign of pain. ${ }^{2}$

Though researchers have provided numerous pain assessment scales for neonates, none of them can be trusted for an error-free pain assessment in neonates practically. These scales are based on the physiological assessments (heart-rate, blood pressure, temperature, vagal tone, oxygen saturation, and palm sweating), behavioural assessment (facial expression, cry vocalization, waking state), and response to painful stimuli. The legitimacy and age-specificity (normal term, pre-term, very pre-term birth) of these pain assessment scales has always remained debatable among the clinical researchers. ${ }^{16}$ Principally, pharmacological and environmental strategies are applied to cope pain in neonates. Drug therapy using analgesics, anaesthetics, and sedatives have clinical efficacy in managing pain among neonates. Practice of using paracetamol and regional anaesthesia to minimize neonatal pain is widely seen among the clinicians. ${ }^{16}$ Though, prolonged usage, side-effects, and possible drug dependency always remain a concern with using drug therapy. Presently clinicians have shown an inclination towards the use of non-pharmacological or environmental strategies to cope pain in neonates. These includes; sucrose, swaddling, co-bedding of twins, facilitated tucking, nonnutritive sucking, kangaroo care, breast feeding. ${ }^{17}$ Parents are also supportive for usage of environmental strategies for managing pain 
and stress in their neonates. Currently sucrose is one of the most widely used non-pharmacological methods to reduce neonatal pain. ${ }^{18}$ Kangaroo Care or skin-to-skin contact between mother and neonate is another method that has proven its clinical efficacy in reducing neonatal pain. ${ }^{19}$ Among twin neonates, positive effects of co-bedding are seen while managing procedural pain like pain after heel lance. ${ }^{20}$ Positive parent interaction is yet another effective measure that is found to be helpful in comforting neonates from procedural pain and stress. ${ }^{18}$

Pain in neonates though remains unidentified, lead to adverse physical, emotional, and behavioural outcomes later in life. It has been observed that even though, clinicians are knowledgeable about the neonatal pain, yet a gap does exist between the knowledge and evidence-based practice for management of neonatal pain. Validated research evidences about the efficacy of the pain management scale and clinical guidelines could possibly bridge this gap. Timely screening and effective pain management strategies hold the potential to prevent accumulation of adverse acute and long-term effects among neonates. Thereby, improving the prospective physiological, emotional, and behavioural growth of the neonate. Effective and prompt pain management strategies also reduce the sufferings and improve the quality of life of the neonate. Hence, it is very important to identify, screen and manage the signs of pain and stress among neonates.

\section{Acknowledgements}

None.

\section{Conflicts of interest}

The author declares that there is no conflict of interest.

\section{Funding}

None.

\section{References}

1. Als H, Duffy FH, McAnulty GB, et al. Early experience alters brain function and structure. Paediatrics. 2004;113(4):846-857.

2. Bonan KC, Pimentel Filho Jda C, Tristao RM, et al. Sleep deprivation, pain and prematurity: a review study. Arq Neuropsiquiatr. 2015;73(2):147-154.

3. WHO: recommended definitions, terminology and format for statistical tables related to the perinatal period and use of a new certificate for cause of perinatal deaths. Modifications recommended by FIGO as amended October 14, 1976. Acta Obstet Gynecol Scand. 1973;56(3):247-253.

4. Blencowe H, Cousens S, Chou D, et al. Born to soon: the global epidemiology of fifteen million pre-term birth. Reprod Health 2013;10(suppl 1):S2.
5. Vinall J, Grunau RE. Impact of repeated procedural pain-related stress in infants born very preterm. Pediatr Res. 2014;75(5):584-587.

6. Grunau RE. Neonatal pain in very preterm infants: long-term effects on brain, neurodevelopment and pain reactivity. Rambam Maimonides Med J. 2013;4(4):e0025.

7. Fitzgerald M, Shaw A, MacIntosh N. Postnatal development of the cutaneous flexor reflex: comparative study of preterm infants and newborn rat pups. Dev Med Child Neurol. 1988;30(4):520-526.

8. Rodrigues AC, Guinsburg R. Pain evaluation after a non-nociceptive stimulus in preterm infants during the first 28 days of life. Early Hum Dev. 2013;89(2):75-79.

9. Hatfield LA. Neonatal pain: What's age got to do with it? Surg Neurol Int. 2014;5(Suppl 13):S479-S489.

10. Vinall J, Grunau R, Bjornson B. Impact of neonatal pain-related stress on brain and IQ at school age in children born preterm. pediatrics, poster presentation, 9th international forum on pediatric pain; White point, NS, Canada. 2013.

11. Doesburg SM, Chau CM, Cheung TP, et al. Neonatal pain-related stress, functional cortical activity and visual-perceptual abilities in school-age children born at extremely low gestational age. Pain. 2013;154(10):1946-1952.

12. Grunau RE, Whitfield MF, Petrie-Thomas J, et al. Neonatal pain, parenting stress and interaction, in relation to cognitive and motor development at 8 and 18 months in preterm infants. Pain. 2009;143(12):138-146.

13. Anand KJ, International evidence-based group for neonatal pain. Consensus statement for the prevention and management of pain in the new-born. Arch Pediatr Adolesc Med. 2001;155(2):173-180.

14. Lago P, Garetti E, Merazzi D, et al. Guidelines for procedural pain in the new-born. Acta Paediatr. 2009;98(6):932-939.

15. Coughlin M, Gibbins S, Hoath S. Core measures for developmentally supportive care in neonatal intensive care units: theory, precedence and practice. J Adv Nurs. 2009;65(10):2239-2248.

16. Maxwell LG, Malavolta CP, Fraga MV. Assessment of pain in the neonate. Clin Perinatol. 2013;40(3):457-469.

17. Pillai Riddell RR, Racine NM, Turcotte K, et al. Non-pharmacological management of infant and young child procedural pain. Cochrane Database Syst Rev. 2011;5(10):CD006275.

18. Walker SM. Neonatal pain. Paediatric Anaesthesia. 2014;24(1):39-48.

19. Johnston CC, Stevens B, Pinelli J, et al. Kangaroo care is effective in diminishing pain response in preterm neonates. Arch Pediatr Adolesc Med. 2003;157(11):1084-1088.

20. Campbell-Yeo ML, Johnston CC, Joseph K, et al. Co-bedding as a comfort measure for twins undergoing painful procedures (CComForT Trial). BMC Pediatr. 2009;9:76. 